\title{
Analytical solutions for energy spectra of electrons accelerated by nonrelativistic shock-waves in shell type supernova remnants
}

\author{
V. N. Zirakashvili ${ }^{1,2}$ and F. Aharonian ${ }^{2,3}$
}

\author{
${ }^{1}$ Pushkov Institute of Terrestrial Magnetism, Ionosphere and Radiowave Propagation, 142190 Troitsk, Moscow Region, Russia \\ e-mail: zirak@izmiran.ru \\ 2 Max-Planck-Institut für Kernphysik, Postfach 103980, 69029 Heidelberg, Germany \\ 3 Dublin Institute for Advanced Studies, 5 Merrion Square, Dublin 2, Ireland
}

Received 2 October 2006 / Accepted 12 January 2007

\begin{abstract}
Context. Recent observations of hard X-rays and very high energy gamma-rays from a number of young shell type supernova remnants indicate the importance of detailed quantitative studies of energy spectra of relativistic electrons formed via diffusive shock acceleration accompanied by intense nonthermal emission through synchrotron radiation and inverse Compton scattering.

Aims. The aim of this work was derivation of exact asymptotic solutions of the kinetic equation which describes the energy distribution of shock-accelerated electrons for an arbitrary energy-dependence of the diffusion coefficient.

Methods. The asymptotic solutions at low and very high energy domains coupled with numerical calculations in the intermediate energy range allow analytical presentations of energy spectra of electrons for the entire energy region.

Results. Under the assumption that the energy losses of electrons are dominated by synchrotron cooling, we derived the exact asymptotic spectra of electrons without any restriction on the diffusion coefficient. We also obtained simple analytical approximations which describe, with accuracy better than ten percent, the energy spectra of nonthermal emission of shock-accelerated electrons due to the synchrotron radiation and inverse Compton scattering.

Conclusions. The results can be applied for interpretation of X-ray and gamma-ray observations of shell type supernova remnants, as well as other nonthermal high energy source populations like microquasars and large scale synchrotron jets of active galactic nuclei.
\end{abstract}

Key words. acceleration of particles - radiation mechanism: non-thermal - shock waves - ISM: supernova remnants

\section{Introduction}

The nonthermal X-ray emission detected from a number of young shell-type SNRs (for a review see e.g. Vink 2006) is generally interpreted as synchrotron radiation of relativistic electrons accelerated diffusively by shock waves to multi-TeV energies (see e.g. Drury et al. 2001). Two other (alternative) radiation mechanisms - the bremsstrahlung of subrelativistic electrons and the inverse Compton (IC) scattering of moderately relativistic electrons - are not sufficiently effective to explain the observed X-ray fluxes. The discovery of $\mathrm{TeV}$ gamma-rays from young SNRs, in particular from Cas A (Aharonian et al. 2001), RX J1713.7-3946 (Enomoto et al. 2002; Aharonian et al. 2004) and RX J0852.0-4622 (Katagiri et al 2005; Aharonian et al. 2005) provides unambiguous evidence of acceleration of particles (electrons and/or protons) to energies $100 \mathrm{TeV}$ and beyond. Unfortunately even the very high quality morphological and spectrometric studies of young SNRS in TeV gamma-rays performed with the HESS array of imaging atmospheric Cherenkov telescopes do not allow robust conclusions concerning the origin of gamma-rays. For example, in the case of the best studied gamma-ray emitting SNR, RX J1713.7-3946, the hadronic model can explain satisfactorily both the overall energetics and the spectral features of $\mathrm{TeV}$ gamma-ray emission. Nevertheless, the inverse Compton origin of gamma-rays remains an alternative option, provided that the magnetic field in the gammaray production region does not exceed $10 \mu \mathrm{G}$ (Aharonian et al. 2006).
Power-law distributions of relativistic particles are readily formed at astrophysical shocks, in particular in young shell type SNRs, through the so-called diffusive shock acceleration (DSA) mechanism (Krymsky 1977; Bell 1978; Axford et al. 1977). The maximum energy of accelerated particles and the shape of the spectrum around and beyond the maximum energy (the so-called "cutoff region") is determined by the competition between the acceleration and escape rates, as well as, in the case of electrons, by radiative (synchrotron and inverse Compton) energy losses. In this regard, the most important information about the accelerator is contained in the energy distribution of particles in the cutoff region. The spectrum of relativistic electrons in the cutoff region is formed under conditions when particles gain energy during the shock crossing and lose energy simultaneously. In young SNRs, the radiative cooling of electrons results in formation of highest energy tails of synchrotron X-rays and inverse Compton gamma-rays, at $\geq 1 \mathrm{keV}$ and $\geq 10 \mathrm{TeV}$, respectively. Thus, the comparison of hard X-ray and $\mathrm{TeV}$ gamma-ray observations with the spectral features predicted by the DSA model in these energy bands gives direct information about the key parameters characterizing the process of particle acceleration. In this regard, analytical presentations of the energy distributions of high energy electrons and the spectra of their synchrotron and IC radiation components provide effective tools for studies of nonthermal processes in SNRs.

So far, analytical solutions for the electron spectra at the plane shock have been derived only for a special case of energyindependent diffusion coefficient (Bulanov \& Dogiel 1979; 


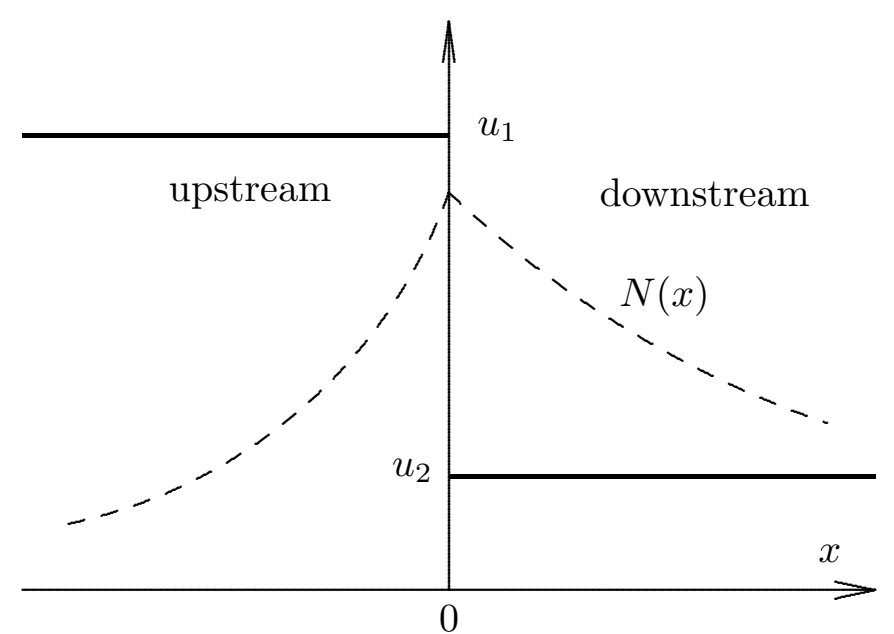

Fig. 1. The shock speeds in the upstream and downstream regions (solid lines) and the spatial distributions of electrons (dashed lines), accelerated at the plane shock.

Webb et al. 1984; Heavens \& Meisenheimer 1987). At the same time, for typical shock speeds of several thousand $\mathrm{km} \mathrm{s}^{-1}$ in young SNRs, the particles, in particular electrons, have a chance to be boosted to multi-TeV energies only if the acceleration proceeds in the most effective way, the so-called Bohm diffusion limit. This implies strong energy-dependence of the diffusion coefficient, $D(E) \propto E$. Therefore, it is important to derive analytical solutions for the electron spectra for a more realistic case of diffusion.

In this paper we present exact asymptotic solutions for the high-energy tails of distributions of electrons formed at the plane shock, assuming arbitrary energy-dependencies of both the diffusion coefficient and the energy lose rate of electrons. The spectrum of electrons in the low-energy domain where the radiative cooling can be neglected, is well known from the solution of the transport equation characterizing the acceleration at the shock without losses. On the other hand, assuming that the cooling of electrons at very high energies is dominated by synchrotron losses, we obtained simple analytical expressions for the exact asymptotic solutions in the high energy domain. In order to extend the analytic presentations to a broader energy range, we should "glue" the asymptotic solutions applicable at very low and very high energies, using numerical calculations performed at intermediate energies. We treat separately the electron distributions at the shock, as well as downstream and upstream of the shock, and calculate the spectra of synchrotron and IC radiation in these regions. We present these spectra in forms of simple analytical approximations which appear significantly different from ones often used in the literature for the fits of spectral measurements and subsequent interpretations of the $\mathrm{X}$-ray and $\mathrm{TeV}$ gamma-ray observations of SNRs in terms of synchrotron and $\mathrm{IC}$ radiation of multi- $\mathrm{TeV}$ electrons.

\section{Asymptotic form of the electron spectra}

Below we consider acceleration of electrons by the plane shock. The upstream plasma moves with a speed $u=u_{1}$ towards the shock from $-\infty$ of the $x$ axis (see Fig. 1). Then, the downstream speed is $u_{2}=u_{1} / \sigma$, where $\sigma$ is the shock compression ratio. The electron momentum distribution $N(p, x)$ obey the following equation written in the shock frame upstream and downstream of the shock:

$\frac{\partial}{\partial x} D \frac{\partial N}{\partial x}-u \frac{\partial N}{\partial x}+\frac{1}{p^{2}} \frac{\partial}{\partial p} p^{2} b(p) N=0$.

Here $b(p)$ is the energy lose rate of particles $(b>0)$. Hereafter we assume that the synchrotron losses dominate over the escape of electrons from the system. The electron momentum distribution $N(p)$ is normalized to $n=4 \pi \int p^{2} \mathrm{~d} p N(p)$, where $n$ is the number density of accelerated particles.

Boundary condition at the shock front, $x=0$, can be written as

$u_{1} \frac{p}{\gamma_{\mathrm{s}}} \frac{\partial N_{0}}{\partial p}=\left.D_{2} \frac{\partial N}{\partial x}\right|_{x=+0}-\left.D_{1} \frac{\partial N}{\partial x}\right|_{x=-0}$,

where $\gamma_{\mathrm{s}}=3 \sigma /(\sigma-1)$ is the power-law index of particles accelerated at the absence of energy losses, $N_{0}(p)$ is the electron distribution at the shock front, $D_{1}$ and $D_{2}$ are the diffusion coefficients upstream and downstream of the shock, respectively.

Analytical solutions of Eqs. (1) and (2) can be obtained for simplified conditions, e.g. at low energies when the radiative energy losses can be neglected, or in the case of energyindependent diffusion-coefficient. On the other hand, it is possible to derive asymptotic solutions for an arbitrary diffusion coefficient in the very high energy regime, where the energy losses of particles dominate over their acceleration. In this regime, it is convenient to present the solution for the steady-state energy distribution of electrons in the following form:

$N_{1,2}=K_{1,2}(x, p) \exp \left[S_{1,2}(x, p)\right]$

where indices 1, 2 correspond to the upstream and downstream regions, respectively. In the asymptotic regime, when losses dominate over the acceleration, the function $S(x, p)$ obtains large negative values. According to the standard asymptotic method we assume that $S(x, p)$ is proportional to $b(x, p)$. After substitution of Eq. (3) into Eq. (1) and keeping all terms which are proportional to $b^{2}$ and $b$, one finds

$D_{1,2}\left(\frac{\partial S_{1,2}}{\partial x}\right)^{2}+b_{1,2} \frac{\partial S_{1,2}}{\partial p}=0$

$D_{1,2} K_{1,2} \frac{\partial^{2} S_{1,2}}{\partial x^{2}}+2 D_{1,2} \frac{\partial K_{1,2}}{\partial x} \frac{\partial S_{1,2}}{\partial x}$

$-u_{1,2} K_{1,2} \frac{\partial S_{1,2}}{\partial x}+\frac{1}{p^{2}} \frac{\partial}{\partial p} p^{2} b_{1,2} K_{1,2}=0$

Denoting by

$S_{0}(p)=\left.S_{1}(p)\right|_{x=0}=\left.S_{2}(p)\right|_{x=0}$,

$K_{0}(p)=\left.K_{1}(p)\right|_{x=0}=\left.K_{2}(p)\right|_{x=0}$,

and performing the same expansion for the boundary condition given by Eq. (2), we obtain

$\frac{u_{1} p}{\gamma_{\mathrm{s}}} \frac{\partial S_{0}}{\partial p}=\left.D_{2} \frac{\partial S_{2}}{\partial x}\right|_{x=0}-\left.D_{1} \frac{\partial S_{1}}{\partial x}\right|_{x=0}$,

$\frac{u_{1} p}{\gamma_{\mathrm{s}}} \frac{\partial K_{0}}{\partial p}=\left.D_{2} \frac{\partial K_{2}}{\partial x}\right|_{x=0}-\left.D_{1} \frac{\partial K_{1}}{\partial x}\right|_{x=0}$ 
The derivatives of functions $S_{1}$ and $S_{2}$ on $x$ and the derivative $\partial S_{0} / \partial p$ can be found from Eqs. (4) and (8):

$$
\begin{aligned}
& \left.\frac{\partial S_{1}}{\partial x}\right|_{x=0}=\frac{\gamma_{\mathrm{s}}}{u_{1} p} \sqrt{\frac{b_{1}}{D_{1}}}\left(\sqrt{b_{1} D_{1}}+\sqrt{b_{2} D_{2}}\right), \\
& \left.\frac{\partial S_{2}}{\partial x}\right|_{x=0}=-\frac{\gamma_{\mathrm{s}}}{u_{1} p} \sqrt{\frac{b_{2}}{D_{2}}}\left(\sqrt{b_{1} D_{1}}+\sqrt{b_{2} D_{2}}\right), \\
& \frac{\partial S_{0}}{\partial p}=-\frac{\gamma_{\mathrm{s}}^{2}}{u_{1}^{2} p^{2}}\left(\sqrt{b_{1} D_{1}}+\sqrt{b_{2} D_{2}}\right)^{2} .
\end{aligned}
$$

The solution of the last equation is

$$
S_{0}=-\frac{\gamma_{\mathrm{s}}^{2}}{u^{2}} \int_{0}^{p} \frac{\mathrm{d} p^{\prime}}{p^{\prime 2}}\left(\sqrt{b_{1}\left(p^{\prime}\right) D_{1}\left(p^{\prime}\right)}+\sqrt{b_{2}\left(p^{\prime}\right) D_{2}\left(p^{\prime}\right)}\right)^{2} \text {. }
$$

Taking the first derivative of Eq. (4) on $x$, and using Eqs. (10) and (11) we find

$$
\begin{aligned}
& \left.\frac{\partial^{2} S_{1}}{\partial x^{2}}\right|_{x=0}=-\frac{b_{1}}{2 D_{1}} \frac{\partial}{\partial p} \ln \sqrt{\frac{b_{1}}{p^{2} D_{1}}}\left(\sqrt{b_{1} D_{1}}+\sqrt{b_{2} D_{2}}\right), \\
& \left.\frac{\partial^{2} S_{2}}{\partial x^{2}}\right|_{x=0}=-\frac{b_{2}}{2 D_{2}} \frac{\partial}{\partial p} \ln \sqrt{\frac{b_{2}}{p^{2} D_{2}}}\left(\sqrt{b_{1} D_{1}}+\sqrt{b_{2} D_{2}}\right) .
\end{aligned}
$$

After substitution of these functions into Eq. (5), and using Eq. (9), we arrive at the following ordinary differential equation

$p \frac{\partial K_{0}}{\partial p} K_{0}^{-1}=-\frac{1}{2}+\frac{\sqrt{D_{2}} p \frac{\partial}{\partial p} \sqrt{b_{2}}+\sqrt{D_{1}} p \frac{\partial}{\partial p} \sqrt{b_{1}}}{\sqrt{b_{1} D_{1}}+\sqrt{b_{2} D_{2}}}$,

the solution of which can be written in the following form:

$$
K_{0}(p) \propto p^{-1 / 2} \exp \int \mathrm{d} p \frac{\sqrt{D_{2}} \frac{\partial}{\partial p} \sqrt{b_{2}}+\sqrt{D_{1}} \frac{\partial}{\partial p} \sqrt{b_{1}}}{\sqrt{b_{1} D_{1}}+\sqrt{b_{2} D_{2}}} .
$$

Equations (3), (13) and (17) determine the asymptotic form of the electron spectrum at the shock front $N_{0}(p)$ for an arbitrary diffusion coefficient. In the case of an energy-independent diffusion, it coincides with the exact solution derived by Webb et al. (1984).

These expressions can be significantly simplified in the case of the same energy-dependence of the diffusion coefficient and the energy lose rate upstream and downstream: $b_{2}=b=\xi b_{1}$, $D_{2}=D=\kappa D_{1}$. In this case

$K_{0}(p) \propto \sqrt{b / p}$,

and Eq. (3) is reduced to

$$
N_{0} \propto \sqrt{\frac{b}{p}} \exp \left[-\frac{\gamma_{\mathrm{s}}^{2}}{u_{1}^{2}}\left(1+\frac{1}{\sqrt{\xi \kappa}}\right)^{2} \int_{0}^{p} \frac{\mathrm{d} p^{\prime}}{p^{\prime 2}} b\left(p^{\prime}\right) D\left(p^{\prime}\right)\right] .
$$

The integrations of $F_{1}=\int_{-\infty}^{0} \mathrm{~d} x N(x, p)$ and $F_{2}=\int_{0}^{\infty} \mathrm{d} x N(x, p)$ give the integrated electron spectra $F_{1}(p)$ and $F_{2}(p)$ in the upstream and downstream regions, respectively. They are of great practical interest in many astrophysical situations. At large momenta they are determined by the ratios of the electron spectrum at the shock front to the absolute values of derivatives given by Eqs. (10) and (11):

$$
F_{1}(p)=\frac{\xi}{1+\sqrt{\kappa \xi}} \frac{u_{1} p}{\gamma_{\mathrm{s}} b(p)} N_{0}(p)
$$

$F_{2}(p)=\frac{\sqrt{\kappa \xi}}{1+\sqrt{\kappa \xi}} \frac{u_{1} p}{\gamma_{\mathrm{s}} b(p)} N_{0}(p)$

Equations (19)-(21) describe the form of the spectrum at large energies. At small energies the losses are negligible and the electron spectrum is power-law with an index $\gamma_{\mathrm{s}}$. In order to describe the transition between this two extreme regimes, Eq. (1) has been solved numerically using an implicit finite-difference scheme.

\subsection{The case of Bohm diffusion}

Below we consider the most interesting case which assumes that the diffusion of electrons proceeds in the so-called Bohm diffusion regime and the energy losses of electrons are dominated by synchrotron radiation. We present the diffusion coefficient in the form $D=\eta c r_{\mathrm{g}} / 3$, where $r_{\mathrm{g}}=p c / q B$ is the gyroradius of particles, $B$ is the magnetic field strength, $q$ and $m$ are the electric charge and mass of the electron, respectively. The factor $\eta \geq 1$ allows deviation of the diffusion coefficient from its minimum value $\eta=1$ (the nominal Bohm diffusion). The synchrotron loss rate averaged over the pitch angles is $b(p)=4 q^{4} B^{2} p^{2} / 9 m^{4} c^{6}$. Since the Bohm diffusion and synchrotron losses are determined by the magnetic field strength, the parameter $\xi=\kappa^{-2}$, where $\kappa$ is the ratio of the magnetic field upstream to the magnetic field downstream, $\kappa=B_{1} / B_{2}$. Equation (19) shows that in the high energy cut-off region the spectrum has a "Gaussian" type behavior, $N_{0} \propto \exp \left(-p^{2} / p_{0}^{2}\right)$, where

$$
\begin{aligned}
& \frac{p_{0}}{m c}=\left(1+\kappa^{1 / 2}\right)^{-1} \frac{m c u_{1}}{\gamma_{\mathrm{s}} \sqrt{2 \eta q^{3} B / 27}}= \\
& \frac{2.86 \times 10^{8}}{\gamma_{\mathrm{s}} \eta^{1 / 2}\left(1+\kappa^{1 / 2}\right)}\left(\frac{u_{1}}{3000 \mathrm{~km} \mathrm{~s}^{-1}}\right)\left(\frac{B}{100 \mu \mathrm{G}}\right)^{-1 / 2} .
\end{aligned}
$$

Here the shock speed $u_{1}$ and the magnetic field in the downstream region $B_{2}=B$ are normalized to $3000 \mathrm{~km} \mathrm{~s}^{-1}$, and $100 \mu \mathrm{G}$, which are quite typical for shells of young SNRs.

At $p=p_{0}$, the characteristic lifetime of electrons due to the synchrotron cooling is

$\tau=p_{0} / b\left(p_{0}\right)=8.6 \gamma_{\mathrm{s}}\left(1+\kappa^{1 / 2}\right) \eta^{1 / 2}$

$\times\left(\frac{u_{1}}{3000 \mathrm{~km} \mathrm{~s}^{-1}}\right)^{-1}\left(\frac{B}{100 \mu \mathrm{G}}\right)^{-3 / 2} \mathrm{yr}$.

The synchrotron losses have a strong impact on formation of the energy spectrum of electrons at energies for which this time is small compared to the characteristic dynamical times of the source, e.g. the age of the accelerator. If this condition is fulfilled, the electrons with such energies are concentrated in the shock vicinity, and therefore the plane shock approximation is well justified.

In the case of standard shock acceleration in the Bohm diffusion regime with $\kappa=1$ and $\gamma_{\mathrm{s}}=4$, we combined the asymptotic analytical solutions, obtained at low and very high energies, with numerical calculations performed in the transition region. This allows a simple analytical presentation of the electron spectrum at the shock over the entire energy range:

$N_{0}(p) \propto p^{-4}\left[1+0.66\left(p / p_{0}\right)^{5 / 2}\right]^{9 / 5} \exp \left(-p^{2} / p_{0}^{2}\right)$

The spectrum of electrons given by Eq. (24) is shown in Fig. 2. At low and large energies Eq. (24) coincides with 


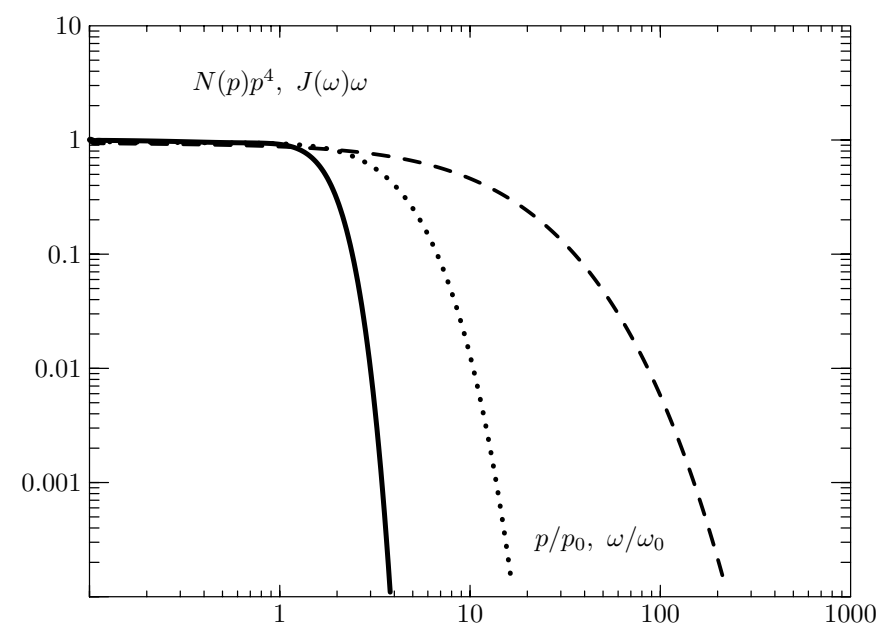

Fig. 2. The electron distribution at the shock front (solid line) and the spatially integrated spectrum of synchrotron radiation (dashed line) produced by electrons accelerated at the shock with compression ratio $\sigma=4$ and equal upstream and downstream magnetic fields $(\kappa=1)$. The spectrum of synchrotron radiation obtained using the $\delta$-function approximation is also shown (dotted line).

the exact asymptotic solutions: $N_{0}(p) \propto p^{-4}$ and $N_{0}(p) \propto$ $p^{1 / 2} \exp \left(-p^{2} / p_{0}^{2}\right)$, respectively. In the cutoff region, the spectrum is proportional to the product of two terms - a powerlaw term with a positive slop $\left(p^{1 / 2}\right)$ and an exponential term, $\exp \left(-p^{2} / p_{0}^{2}\right)$. While the first term can be interpreted as a pileup $^{1}$, it in fact does not sow-up because the second (exponential) term effectively cancels this feature. As a result, an almost perfect power-law ( $p^{-4}$ type) spectrum is formed up to $p \sim p_{0}$, with a super-exponential cutoff afterwards (see Fig. 2).

It is easy to relate, using Eq. (1) and boundary condition given by Eq. (2) the integrated spectra in downstream, upstream and the spectrum at the shock:

$F_{2}+\kappa^{2} F_{1}=l \frac{p_{0}}{p} N_{0}(p)$, and $l=\frac{u_{1} p^{2}}{\gamma_{\mathrm{s}} b p_{0}}$.

This relation is valid for all energies. It implies that for $\kappa=1$ (i.e. equal magnetic field strengths upstream and downstream) the integrated spectrum $F=F_{1}+F_{2} \propto N_{0} p^{-1}$.

The case of different magnetic fields in the downstream and upstream regions, more specifically, for the scenario when the magnetic field downstream is stronger than magnetic field upstream by a factor of $\kappa^{-1}=\sqrt{11}$, is of practical interest, in particular for nonthermal emission of young supernova remnants. This case, which corresponds to the increase of the isotropic random B-field at the shock with the "standard" compression factor $\sigma=4$, can be described by the following analytical approximations for the electron distributions at the shock front:

$N_{0}(p) \propto\left(p / p_{0}\right)^{-4}\left[1+0.523\left(p / p_{0}\right)^{9 / 4}\right]^{2} \exp \left(-p^{2} / p_{0}^{2}\right)$,

and in the upstream region

$F_{1}(p) \propto 0.70 l\left(p / p_{0}\right)^{-3}\left[1+1.7\left(p / p_{0}\right)^{3}\right]^{5 / 6} \exp \left(-p^{2} / p_{0}^{2}\right)$.

For the downstream, the integrated energy spectrum of electrons can be found from Eq. (25):

$F_{2}(p)=N_{0}(p) l \frac{p_{0}}{p}-0.09 F_{1}(p)$.

1 The possible appearance of a pile-ups in the electron spectra within the so-called "box" model of shock acceleration has been noticed by Drury et al. (1999).

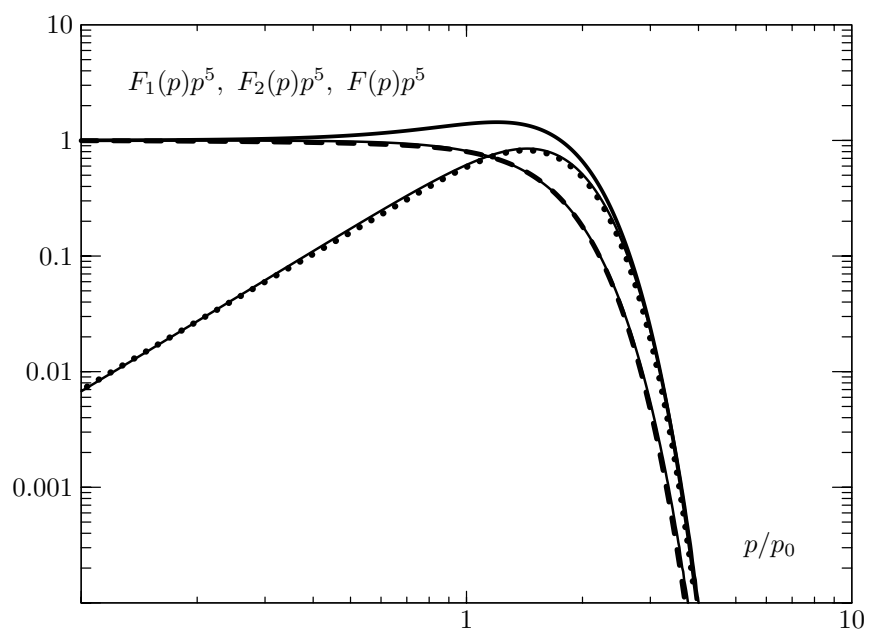

Fig. 3. The integrated upstream $\left(F_{1}\right.$; dotted line) and downstream $\left(F_{2}\right.$; dashed line) electron spectra given by Eqs. (27), (28), as well as the overall $F=F_{1}+F_{2}$ spectrum (solid line) for the shock with compression ratio $\sigma=4$ and the ratio of the magnetic fields downstream and upstream $k^{-1}=\sqrt{11}$. The spectra $F_{1}$ and $F_{2}$ obtained numerically are also shown (thin solid lines).

Note that the spectrum integrated over the upstream region, at small energies is very flat, $F_{1} \propto p N_{0}(p) \propto p^{-3}$. This has a simple explanation related to the spatial scale of the electron distribution; in the upstream region it is proportional to the diffusion coefficient.

The integrated spectra of electrons in the upstream and downstream regions given by Eqs. (26)-(28) as well as results obtained numerically are shown in Fig. 3.

The dependence of the spectra given by Eqs. (24), (26)-(28) on the factor $\eta$ is expressed through the momentum $p_{0}$.

\section{Synchrotron radiation}

The synchrotron emissivity of electrons is determined as

$\epsilon(\omega)=\frac{\sqrt{3} B q^{3}}{2 \pi m c^{2}} \int p^{2} \mathrm{~d} p N(p) R\left(\omega / \omega_{\mathrm{c}}\right)$

where $\omega_{\mathrm{c}}$ is the characteristic frequency of synchrotron radiation $\omega_{\mathrm{c}}=1.5 \mathrm{qB} p^{2} / \mathrm{m}^{3} \mathrm{c}^{3}$. The function $R$ describes synchrotron radiation of a single electron in magnetic field with chaotic directions. Crusius \& Schlickeiser (1986) derived an exact expression for $R\left(\omega / \omega_{\mathrm{c}}\right)$ in terms of Whittaker's function. With an accuracy of several percent $R\left(\omega / \omega_{\mathrm{c}}\right)$ can be presented in a simple analytical form

$R\left(\omega / \omega_{\mathrm{c}}\right)=\frac{1.81 \exp \left(-\omega / \omega_{\mathrm{c}}\right)}{\sqrt{\left(\omega_{\mathrm{c}} / \omega\right)^{2 / 3}+(3.62 / \pi)^{2}}}$.

These functions are compared in Fig. 4.

The energy flux $\left(J(\omega)=\omega \mathrm{d} N_{\text {rad }} / \mathrm{d} \omega\right)$ of synchrotron radiation produced at the shock is determined by the integral of the emissivity along the line of sight $l$ and over the solid angle $\Omega$ $J(\omega)=\int \mathrm{d} l \mathrm{~d} \Omega \epsilon$. This means that it is determined by the integrated spectrum $F(p)$.

The integration of Eq. (29), using Eqs. (24) and (30), results in the energy flux of radiation $J(\omega)=$ $\int \mathrm{d} p a(p, \omega) \exp (-g(p, \omega))$, where $g(p, \omega)=\omega / \omega_{\mathrm{c}}+p^{2} / p_{0}^{2}$, $a(p, \omega) \propto p^{2} F(p) R\left(\omega / \omega_{\mathrm{c}}\right) \exp (g(p, \omega))$. At large frequencies, when the argument of the exponent is large this integral is 


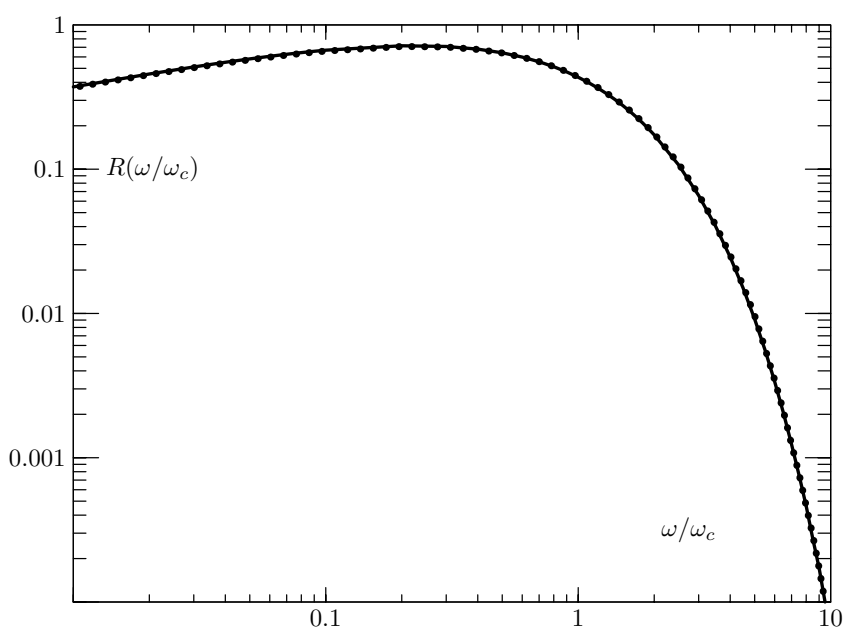

Fig. 4. Comparison of the function $R\left(\omega / \omega_{\mathrm{c}}\right)$ tabulated by Crusius \& Schlickeiser (1986) (dotted line) and the function given by the approximate expression (30) (solid line).

mostly determined by the momentum interval around the momentum $p_{*}$ which minimizes the function $g(p, \omega)$. Thus at large frequencies the energy flux of radiation can be written as $J(\omega)=a\left(p_{*}, \omega\right) \exp \left(-g\left(p_{*}, \omega\right)\right) \sqrt{2 \pi / g^{\prime \prime}\left(p_{*}, \omega\right)}$. Here $g^{\prime \prime}$ is the second derivative of $g$ on $p$. The momentum $p_{*}$ is given by the expression:

$$
\begin{aligned}
& \frac{p_{*}}{m c}=\left(9 \frac{\omega m^{3} c^{3} u_{1}^{2}}{\eta \gamma_{\mathrm{s}}^{2} q^{4} B^{2}}\right)^{1 / 4} \frac{1}{\sqrt{1+\kappa^{1 / 2}}}=8.3 \times 10^{7} \\
& \times \frac{(\hbar \omega / 1 \mathrm{keV})^{1 / 4}}{\gamma_{\mathrm{s}}^{1 / 2} \eta^{1 / 4} \sqrt{1+\kappa^{1 / 2}}}\left(\frac{u_{1}}{3000 \mathrm{~km} \mathrm{~s}^{-1}}\right)^{1 / 2}\left(\frac{B}{100 \mu \mathrm{G}}\right)^{-1 / 2} .
\end{aligned}
$$

Note that at the fixed $\omega$, the characteristic frequency of the synchrotron radiation $\omega_{\mathrm{c}}$ corresponding to $p_{*}$, is smaller than $\omega$.

In the regime of Bohm diffusion the energy flux of synchrotron radiation has an asymptotic form

$J \propto \omega^{3 / 8} \exp \left(-\sqrt{\omega / \omega_{0}}\right)$,

where

$\omega_{0}=\frac{81}{16\left(1+\kappa^{1 / 2}\right)^{2}} \frac{u_{1}^{2} m c}{\eta \gamma_{\mathrm{s}}^{2} q^{2}}$,

or for the corresponding energy of synchrotron photons

$\epsilon_{0}=\hbar \omega_{0}=\frac{2.2 \mathrm{keV}}{\eta\left(1+\kappa^{1 / 2}\right)^{2}}\left(\frac{u_{1}}{3000 \mathrm{~km} \mathrm{~s}^{-1}}\right)^{2} \frac{16}{\gamma_{\mathrm{s}}^{2}}$.

At small energies the integrated downstream synchrotron spectrum is proportional to $\omega^{-\frac{\gamma_{s}-2}{2}}$. In order to describe analytically the synchrotron spectrum over the entire energy range, which should coincide with the exact asymptotic spectra at low and high energies, we use numerical calculations performed in the intermediate energy range. In particular, in the case of $\gamma_{\mathrm{s}}=4$ and $\kappa=1$, we suggest the following approximate formula

$J(\omega) \propto \frac{\omega_{0}}{\omega}\left[1+0.46\left(\frac{\omega}{\omega_{0}}\right)^{0.6}\right]^{11 / 4.8} \exp \left(-\sqrt{\omega / \omega_{0}}\right)$,

which provides an accuracy better than 10 percent.

The energy spectrum of electrons at the shock $N_{0}(p)$ (see Eqs. (24)), is shown in Fig. 2. Because the cooling length of

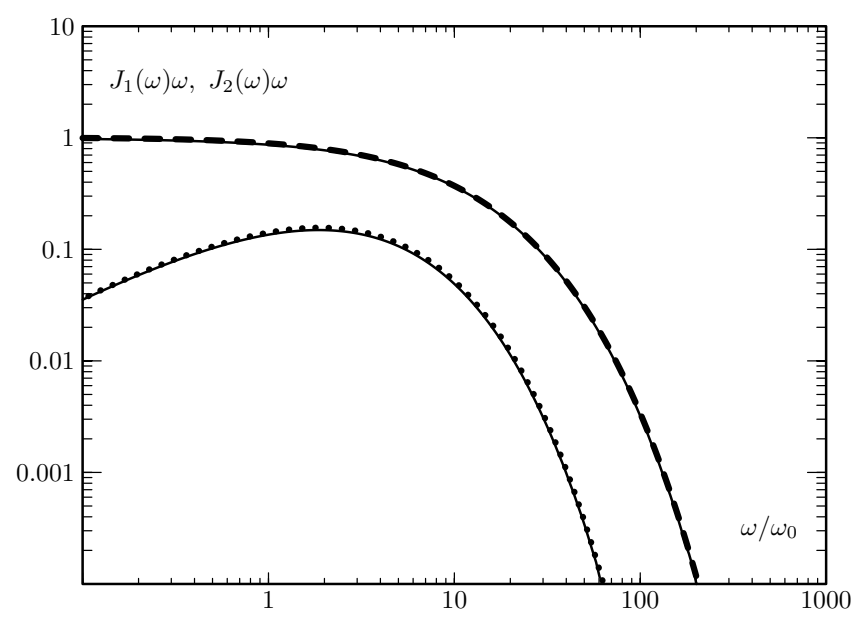

Fig. 5. The energy spectra of synchrotron radiation given by Eqs. (36), (37) and produced by electrons in downstream (dashed line) and upstream (dotted line) regions of the shock with compression ratio $\sigma=4$ and the ratio of the magnetic fields downstream and upstream $\kappa^{-1}=\sqrt{11}$. The corresponding spectra obtained numerically are also shown (solid lines).

electrons downstream the shock is inversely proportional to the momentum, the spatially integrated electron spectrum appears steeper, in particular, in the case of $\kappa=1, F(p)=F_{1}(p)+$ $F_{2}(p) \propto N_{0}(p) / p$. Note that below the cutoff region $F(p) \propto p^{-5}$. The corresponding spectrum of synchrotron radiation of these electrons is shown in Fig. 2. At small frequencies, $J(\omega) \propto \omega^{-1}$.

The synchrotron spectrum obtained using the so-called $\delta$ function approximation is also shown in Fig. 2. It is assumed, that the synchrotron emission of the electron is concentrated at the frequency $\omega=0.29 \omega_{\mathrm{c}}$. This corresponds to the function $R\left(\omega / \omega_{\mathrm{c}}\right) \propto \delta\left(0.29-\omega / \omega_{\mathrm{c}}\right)$. Obviously this approximation does not provide a satisfactory fit.

In the case of $\gamma_{\mathrm{s}}=4, \kappa=1 / \sqrt{11}$ we found the following spectra produced upstream and downstream the shock:

$J_{1}(\omega) \propto 0.58\left[1+1.27\left(\frac{\omega}{\omega_{0}}\right)^{3 / 4}\right]^{1 / 2} \exp \left(-\sqrt{3.32 \frac{\omega}{\omega_{0}}}\right)$

$J_{2}(\omega) \propto \frac{\omega_{0}}{\omega}\left[1+0.38 \sqrt{\frac{\omega}{\omega_{0}}}\right]^{11 / 4} \exp \left(-\sqrt{\frac{\omega}{\omega_{0}}}\right)$.

Note that the spectrum of synchrotron radiation produced upstream the shock at small frequencies is very flat, $J(\omega)=$ const., which reflects the hard electron spectrum in that region (see Eq. (27) and Fig. 3).

The spectra of synchrotron radiation in the upstream and downstream regions given by Eqs. (36) and (37) are shown in Fig. 5. The synchrotron flux in the downstream region is significantly enhanced because of the larger (compressed) magnetic field. The corresponding spectra obtained numerically are also shown.

We should note that the exponential terms in Eqs. (35)-(37) are relatively slow functions (proportional to $\exp \left[-\left(\omega / \omega_{0}\right)^{1 / 2}\right]$ ). Moreover, these exponential terms are multiplied to power-law terms $\left(\omega / \omega_{0}\right)^{s}$ with positive indices, $s \geq 0$, which effectively compensate the exponential terms at frequencies $\omega \sim \omega_{0}$. For that reason the parameter $\omega_{0}$ only formally can be treated as a cutoff frequency. In reality, the breaks (cutoffs) in the synchrotron spectra appear at much higher frequencies, namely at $\omega \geq 10 \omega_{0}$. 
The integrated downstream electron spectrum $F_{2}$ in Fig. 3 and the corresponding synchrotron spectrum $J_{2}$ in Fig. 5 may be applied to the supernova remnants with ages lager than the cooling time given by Eq. (23), i.e. when the electron spectrum in the cut-off region is determined by the energy losses of electrons. Electrons with energies below cut-off energy are accelerated with power-law spectrum and advected downstream. It was also assumed that these electrons lose all their energy downstream that is of course valid for energies larger than the energy of the electron which has the cooling time comparable with the age of the remnant. For smaller energies a threedimensional geometry of the shock wave should be taken into account. Consequently, the integrated downstream electron spectrum and the corresponding synchrotron radiation spectrum appear flatter compared to the ones shown in Figs. 3, 5.

\subsection{The radiation zones}

The asymptotic solutions derived in Sect. 2 can be used for study of coordinate-dependence of electron distributions. Near the shock front, the spatial distribution of electrons has an exponential dependence. The inverse exponential lengths in the upstream and downstream regions are given by Eqs. (10) and (11). Since the synchrotron radiation is produced mainly by electrons with momentum $p_{*}$, the exponential lengths of emissivity (29) in the upstream $l_{\mathrm{u}}$ and downstream $l_{\mathrm{d}}$ regions of the shock can be found from Eqs. (10), (11) and (31):

$l_{\mathrm{d}}=\frac{9}{4 \sqrt{3\left(1+\kappa^{1 / 2}\right)}}\left(\frac{m^{9} c^{17} u_{1}^{2} \eta}{\omega q^{12} B^{6} \gamma_{\mathrm{s}}^{2}}\right)^{1 / 4}$,

and

$l_{\mathrm{u}}=l_{\mathrm{d}} \kappa^{-5 / 4}$.

In particular, for $\gamma_{\mathrm{s}}=4$ we have

$$
\begin{aligned}
l_{\mathrm{d}}= & \frac{1.4 \times 10^{17} \mathrm{~cm}}{\sqrt{1+\kappa^{1 / 2}}} \eta^{1 / 4}\left(\frac{u_{1}}{3000 \mathrm{~km} \mathrm{~s}^{-1}}\right)^{1 / 2} \\
& \times\left(\frac{B}{100 \mu \mathrm{G}}\right)^{-3 / 2}\left(\frac{\hbar \omega}{1 \mathrm{keV}}\right)^{-1 / 4},
\end{aligned}
$$

for the radiation width in downstream, and a factor of $\kappa^{-5 / 4}$ larger in upstream. Note that the above equations are correct only for large frequencies, $\omega>\omega_{0}$.

The radiation width in downstream (40) is relatively small. This permits to explain the narrow X-ray filaments observed in SNRs and to estimate the magnetic field strength (see e.g. Berezhko et al. 2002; Völk et al. 2005).

\section{Inverse Compton scattering}

In the isotropic photon field, the emissivity of the inverse Compton radiation of an electron with Lorentz factor $\gamma$ is described as (Blumenthal \& Gould 1970)

$$
\begin{gathered}
\epsilon_{\mathrm{IC}}(\omega)=2 \hbar c r_{\mathrm{e}}^{2} \int_{\frac{\omega}{4 \gamma^{2}\left(1-\frac{\hbar \omega}{\gamma m c^{2}}\right)}}^{\infty} \mathrm{d} \omega^{\prime} n\left(\omega^{\prime}\right) \frac{\omega}{4 \gamma^{2} \omega^{\prime}}\left[1-2 \frac{\omega^{2}}{\omega_{\mathrm{c}}^{2}}+\right. \\
\left.\frac{\omega}{\omega_{\mathrm{c}}}\left(1+2 \ln \frac{\omega}{\omega_{\mathrm{c}}}\right)+\frac{\hbar^{2} \omega^{2}}{2 m^{2} c^{4} \gamma^{2}\left(1-\frac{\hbar \omega}{\gamma m c^{2}}\right)}\left(1-\frac{\omega}{\omega_{\mathrm{c}}}\right)\right] .
\end{gathered}
$$

Here $\omega_{\mathrm{c}}=4 \omega^{\prime} \gamma^{2}\left(1-\frac{\hbar \omega}{\gamma m c^{2}}\right), r_{\mathrm{e}}=q^{2} / m c^{2}$ is the classical electron radius and $n(\omega)$ is the energy distribution of the target photons. Hereafter we assume that the target photons are described by a Planckian distribution

$n(\omega)=\frac{\omega^{2} /\left(c^{3} \pi^{2}\right)}{\exp \left(\hbar \omega / \kappa_{\mathrm{B}} T\right)-1}$,

where $T$ is the temperature of radiation and $\kappa_{\mathrm{B}}$ is the Boltzman constant. It is easy to show, using Eqs. (41) and (42), that at large frequencies $\omega \gg \gamma^{2} \kappa_{\mathrm{B}} T / \hbar$ the IC emissivity in the Thompson regime behaves as $\epsilon_{\mathrm{IC}}(\omega) \propto \omega \exp \left(-\hbar \omega / 4 \gamma^{2} \kappa_{\mathrm{B}} T\right)$. At very high energies the integration over the electron energy distribution at the shock and the use of the method described in the Sect. 3, give the following asymptotic form of the IC spectrum in the Thompson limit:

$J^{\mathrm{IC}} \propto \omega^{7 / 8} \exp \left(-\sqrt{\omega / \omega_{\mathrm{b}}}\right)$,

with

$\omega_{\mathrm{b}}=\frac{27}{2 \eta\left(1+\kappa^{1 / 2}\right)^{2}} \frac{\kappa_{\mathrm{B}} T u_{1}^{2} m^{2} c^{2}}{\hbar \gamma_{\mathrm{s}}^{2} q^{3} B}$

or, in terms of energy of gamma-rays, $\epsilon_{\mathrm{b}}=\hbar \omega_{\mathrm{b}}$, we have:

$\epsilon_{\mathrm{b}}=\frac{1.2 \mathrm{TeV}}{\eta\left(1+\kappa^{1 / 2}\right)^{2}}\left(\frac{u_{1}}{3000 \mathrm{~km} \mathrm{~s}^{-1}}\right)^{2} \frac{100 \mu \mathrm{G}}{B} \frac{T}{2.7 \mathrm{~K}} \frac{16}{\gamma_{\mathrm{s}}^{2}}$

As before, it is assumed that synchrotron losses dominate over IC losses, i.e. the energy density of the background radiation $U_{\text {rad }} \ll B^{2} / 8 \pi$. In the case of $2.7 \mathrm{~K}$ CMBR this implies $B \geq$ $3 \mu \mathrm{G}$.

Using the exact asymptotic spectra at low and high energies, and the results of numerical calculations at intermediate energies, we can describe the broad-band energy flux of the inverse Compton (Thompson) scattering by simple analytical expressions. In particular, for $\kappa=1$ one has

$J^{\mathrm{IC}}(\omega) \propto \frac{\omega_{\mathrm{b}}}{\omega}\left[1+0.36\left(\frac{\omega}{\omega_{\mathrm{b}}}\right)^{0.7}\right]^{15 / 5.6} \exp \left(-\sqrt{\frac{\omega}{\omega_{\mathrm{b}}}}\right)$.

For the "standard" scenario of shock acceleration with $\gamma_{\mathrm{s}}=4$ and $\kappa=1 / \sqrt{11}$, we obtain the following IC spectra produced upstream and downstream the shock:

$J_{1}^{\mathrm{IC}}(\omega) \propto 0.20\left[1+0.75\left(\frac{\omega}{\omega_{\mathrm{b}}}\right)^{7 / 8}\right] \exp \left(-\sqrt{\frac{\omega}{\omega_{\mathrm{b}}}}\right)$

$J_{2}^{\mathrm{IC}}(\omega) \propto \frac{\omega_{\mathrm{b}}}{\omega}\left[1+0.31\left(\frac{\omega}{\omega_{\mathrm{b}}}\right)^{0.6}\right]^{25 / 8} \exp \left(-\sqrt{\frac{\omega}{\omega_{\mathrm{b}}}}\right)$.

The spectra given by Eqs. (47) and (48) are shown in Fig. 6. They are derived under the assumption that the Compton scattering occurs in the Thompson regime. However, in many cases one should take into account the Klein-Nishina effect. The importance of this effect in the case of black-body radiation field is determined by the parameter

$$
\begin{aligned}
b_{\mathrm{KN}}= & 4 \frac{\kappa_{\mathrm{B}} T p_{0}}{m^{2} c^{3}}=\frac{0.52}{\gamma_{\mathrm{s}} \eta^{1 / 2}\left(1+\kappa^{1 / 2}\right)} \\
& \times\left(\frac{u_{1}}{3000 \mathrm{~km} \mathrm{~s}^{-1}}\right)\left(\frac{B}{100 \mu \mathrm{G}}\right)^{-1 / 2} \frac{T}{2.7 \mathrm{~K}} .
\end{aligned}
$$




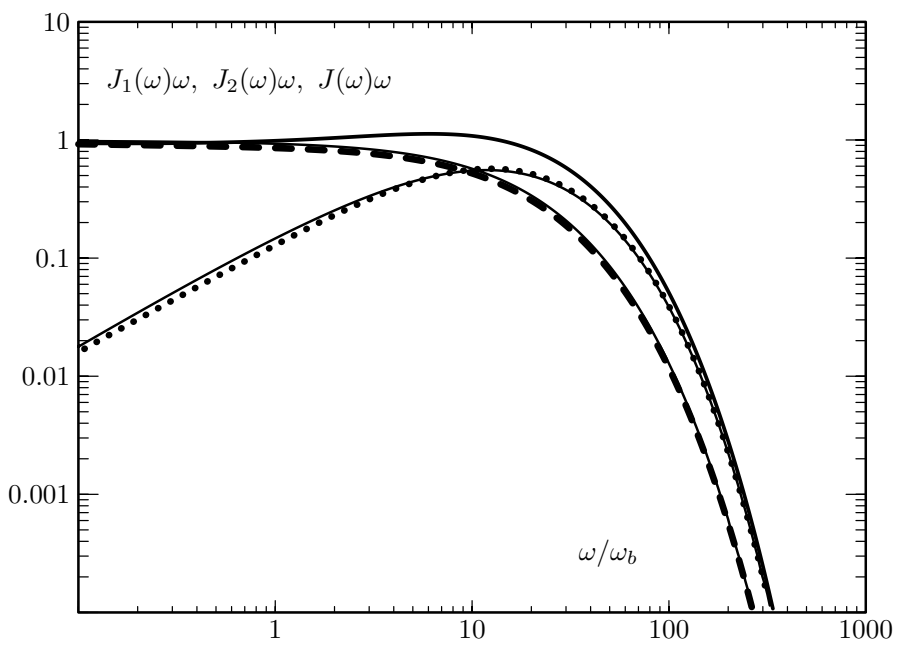

Fig. 6. The spectrum of IC radiation given by Eqs. (47), (48) and produced by electrons accelerated downstream (dashed line) and upstream (dotted line) the shock with compression ratio $\sigma=4$ and the ratio of the magnetic strength downstream and upstream $\sqrt{11}$. The sum $J^{\mathrm{IC}}=J_{1}^{\mathrm{IC}}+J_{2}^{\mathrm{IC}}$ is shown by the solid line. It is assumed that IC scattering proceeds in the Thompson limit, $b_{\mathrm{KN}}=0$, and that synchrotron losses dominate over the IC loses. The corresponding spectra obtained numerically are also shown (thin solid lines).

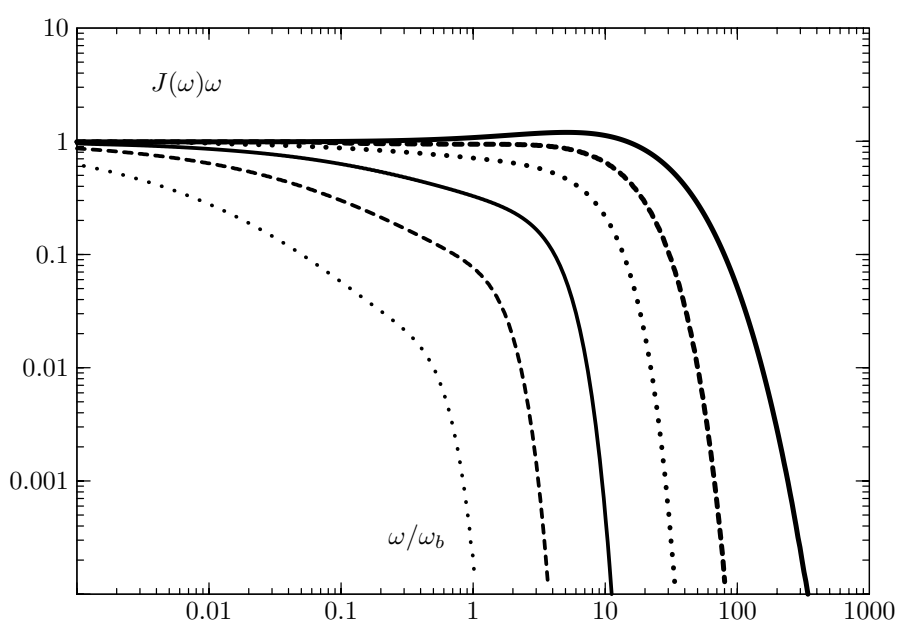

Fig. 7. The spectra of IC radiation produced by electrons accelerated at the shock with compression ratio $\sigma=4$ and the ratio of the magnetic strength downstream and upstream $\sqrt{11}$ for different values of $b_{\mathrm{KN}}: b_{\mathrm{KN}}=0$ (solid line), $b_{\mathrm{KN}}=0.1$ (dashed line), $b_{\mathrm{KN}}=0.3$ (dotted line), $b_{\mathrm{KN}}=1.0$ (thin solid line), $b_{\mathrm{KN}}=3.0$ (thing dashed line) and $b_{\mathrm{KN}}=10.0$ (thin dotted line). The case of dominating synchrotron losses is considered.

The Thompson limit corresponds to $b_{\mathrm{KN}} \ll 1$, while the KleinNishina effect becomes significant already at $b_{\mathrm{KN}} \geq 0.1$. The IC spectra of electrons calculated for different values of the parameter $b_{\mathrm{KN}}$ are shown in Fig. 7.

As long as the energy losses of electrons are dominated by synchrotron radiation, the Klein-Nishina effect makes steeper the spectrum of IC gamma-rays. In particular, it moves the region of the spectral cutoff to lower energies. Below the cutoff region, the energy spectrum of IC gamma-rays still can be described by a (quasi) power-law. The Klein-Nishina effect changes the slope of the gamma-ray spectrum significantly, even for relatively small values of the parameter $b_{\mathrm{KN}}$.

\section{Discussion}

The detection of nonthermal X-ray emission of synchrotron origin clearly demonstrates the existence of multi-TeV electrons in different astrophysical source populations like shell type SNRs - pulsar wind nebulae, microquasars, small and large scale jets in AGN, etc. Given the severe synchrotron losses of electrons, which increase with energy as $\mathrm{d} E / \mathrm{d} t \propto E^{2}$, the acceleration efficiency in these objects should be very high in order to boost the electrons to energies well beyond $1 \mathrm{TeV}$. In particular, in young SNRs with typical shock speeds $u_{1} \sim 2000-3000 \mathrm{~km} \mathrm{~s}^{-1}$, which are established as prominant nonthermal X-ray emitters, the diffusive shock acceleration of electrons should proceed in the regime close to the Bohm diffusion limit, otherwise the synchrotron spectrum would break before achieving the X-ray domain.

Although the basic concepts of diffusive shock acceleration are deeply studied and well understood (see e.g. Malkov \& Drury 2001), many important details remain unexplored. This concerns, in particular the radiation features of the shock accelerated electrons. Over the recent years, several numerical calculations of high energy radiation by particles accelerated in young SNRs have been performed with emphasis on the non-linear effects caused by protons and nuclei on the shock structure (see e.g. Ellison et al. 2000; Berezhko \& Völk 2004).

The shock accelerated electron spectra have been studied analytically (e.g. by Webb et al. 1984), but only for the case of energy-independent diffusion coefficient. Therefore the results of these early works generally cannot be applied to the most interesting sources with synchrotron X-ray emission. These sources, as noticed above, require diffusive shock acceleration of electrons in the regime close to the Bohm diffusion which is characterized by a diffusion coefficient $D(E) \propto E$.

The aim of this paper was a fully analytical treatment of the energy spectra of shock-accelerated electrons and their synchrotron and inverse Compton radiation for an arbitrary energydependent diffusion coefficient. In particular, under the assumption of dominance of synchrotron losses, we derived exact asymptotic solutions in the high energy region. For the energydependent diffusion coefficient written in a rather general form $D \propto p^{\alpha}$, the electron spectrum contains an exponential term $\exp \left[-\left(p / p_{0}\right)^{\beta}\right]$ with $\beta=\alpha+1$ (see Eq. (19)). Thus, only in the case of energy-independent diffusion $(\alpha=0)$ the electron spectrum is characterized by an exponential cutoff. In the most interesting case of Bohm diffusion, the spectral cut-off is of Gaussian type, $\exp \left[-\left(p / p_{0}\right)^{2}\right]$. The corresponding cutoff in the spectrum of syncrotron radiation appears much smoother (see also Fritz 1989 for discussion of this effect). It is described by an exponential term $\exp \left[-\left(\omega / \omega_{0}\right)^{\beta /(2+\beta)}\right]=\exp \left[-\left(\omega / \omega_{0}\right)^{(\alpha+1) /(\alpha+3)}\right]$. For the energy independent diffusion coefficient we have $J(\omega) \propto$ $\exp \left[-\left(\omega / \omega_{0}\right)^{1 / 3}\right]$, while in the case of Bohm diffusion $J(\omega) \propto$ $\exp \left[-\left(\omega / \omega_{0}\right)^{1 / 2}\right]$.

We should note that the $\exp \left[-\left(\omega / \omega_{0}\right)^{1 / 2}\right]$ type behavior of the synchrotron spectrum in the cutoff region formally is similar to the one derived for the generally assumed "power-law with exponential cutoff" electron spectrum, $N_{0}(p) \propto p^{-\Gamma} \exp \left(-p / p_{0}\right)$, and using the $\delta$-function approximation for calculation of the synchrotron radiation (see e.g. Reynolds 1998). However, this is simply a coincidence. First of all, the $\delta$-functional approximation leads to a wrong synchrotron spectrum; the exponential cut-off in the electron spectrum results in the synchrotron spectrum with a $\exp \left(-\omega^{1 / 3}\right)$ type cut-off. More importantly, the spectra of shock accelerated electrons given by Eq. (26)(28) are quite different from the simplified "power-law with 
exponential cutoff" assumption. Correspondingly, because of the dependence $\omega_{\mathrm{c}} \propto p^{2} B$, the effect on the spectrum of synchrotron radiation appears even more pronounced (see Fig. 2).

Exact solutions for electrons are possible only at low and high energies, namely in the energy intervals where $(i)$ the radiative losses of electrons are negligible, and (ii) the radiative energy loss rate exceeds the acceleration rate. The indices of the power-law factors in the asymptotic solutions (19), (32) and (43) significantly differ from the power-law indices of the electrons, as well as the synchrotron and IC spectra below the cutoff regions. In order to connect these solutions we performed numerical calculations in the intermediate energy intervals. This allowed us to obtain, within accuracy better than 10 percent, simple analytical presentations for the spectra of electrons and their synchrotron and inverse Compton radiation components.

Equations (35)-(37) and Eqs. (46)-(48) properly describe the relative contributions of radiation from the regions upstream and downstream the shock. At the same time, they do not allow direct calculations of the absolute synchrotron and inverse Compton luminosities. However, the normalisation constants can be easily calculated from the total energy release through the synchrotron and inverse Compton channels.

It should be noted that in this paper the momentum $p_{0}$ in the electron spectrum, and the corresponding frequencies $\omega_{0}$ and $\omega_{\mathrm{b}}$ which characterize the positions of cut-offs in the spectra of synchrotron and IC radiation components, are found from the exact asymptotic solutions of kinetic equations. Interestingly, the results appear quite close, within a factor of 2, to the estimates derived from the condition "acceleration rate $=$ synchrotron cooling rate". However, as it follows from Figs. 2-6, the real cut-offs appear, in fact, at much higher frequencies. In the case of synchrotron radiation, the spectrum continues without an indication of a break or a cutoff up to $20 \omega_{0}$. The spectrum of inverse Compton gamma-rays turns down also at $\omega \sim 20 \omega_{\mathrm{b}}$ (in the case of constant magnetic field), or it continues up to $\sim 50 \omega_{\mathrm{b}}$ (in the case of different magnetic fields upstream and downstream). The more effective extension of the hard spectrum of IC radiation is explained by the larger spatial scale of electron distribution upstream the shock. This is not important for the synchrotron radiation the contribution of which from the upstream region is suppressed by an order of magnitude. On the other hand, because of the homogeneous distribution of the target radiation field, $2.7 \mathrm{~K}$ $\mathrm{CMBR}$, the IC gamma-ray contribution at highest energies from the upstream region dominates over the contribution from the downstream region.

The results obtained in this paper can be used to fit the X-ray spectra observed from young supernova remnants. The value of the parameter $\omega_{0}$ found from the fitting procedure should be compared with the theoretical value given by Eq. (33). For the known shock velocity of a given supernova remnant, this would allow an important estimate of deviation of the diffusion regime from the extreme Bohm limit. The dependence of the energy spectra on the factor $\eta$ is expressed via parameters $p_{0}, \omega_{0}, \omega_{\mathrm{b}}$. If the width of X-ray filaments is known, the magnetic field strength may be found from Eq. (40). All this in turn would provide reliable estimates of the maximum energy of protons and nuclei accelerated in supernova remnants.

Finally, we note that the results of this paper are applicable for nonrelativistic shocks with velocities up to $u_{1} \sim(0.1-0.3) c$. The derived analytical approximations do not take into account possible nonlinear effects which can somewhat modify the spectra of electrons at low and high energies.

Acknowledgements. V.N.Z. acknowledges the hospitality of the Max-PlanckInstitut für Kernphysik, where this work was carried out. We thank the anonymous referee for many valuable comments.

\section{References}

Aharonian, F. et al. (HEGRA collaboration) 2001, A\&A, 370, 112 Aharonian, F., et al. (HESS collaboration) 2004, Nature, 432, 75 Aharonian, F., et al. (HESS collaboration) 2005, A\&A, 437, L7 Aharonian, F., et al. (HESS collaboration) 2006, A\&A, 449, 223 Axford, W. I., Leer, E., \& Scadron, G. 1977, Proc. 15th Int. Cosmic Ray Conf., Plovdiv, 90, 937

Bell, A. R. 1978, MNRAS, 182, 147

Berezhko, E. G., \& Völk, H. J. 2004, A\&A, 427, 525

Berezhko, E. G., Ksenofontov, L. T., \& Völk, H. J. 2002, A\&A, 395, 943

Blumenthal, G. R., \& Gould, R. J. 1970, Rev. Mod. Phys., 42, 237

Bulanov, S. V., \& Dogiel, V. A. 1979, Sov. Astron. Lett., 5, 278

Crusius, A., \& Schlickeiser, R. 1986, A\&A, 164, L16

Drury, L. O'C., Duffy, P., Eichler, D., \& Mastichiadis, A. 1999, A\&A, 347, 370

Drury, L. O'C., Ellison, D. C., Aharonian, F. A. et al. 2001, Space Sci. Rev., 99, 329

Enomoto, R., Tanimori, T., Naito, T., et al. 2002, Nature, 416, 823

Ellison, D. C., Berezhko, E. G., \& Baring, M. G. 2000, ApJ, 540, 292

Fritz, K. D. 1989, A\&A, 214, 14

Heavens, A. F., \& Meisenheimer, K. 1987, MNRAS, 225, 335

Katagiri, H., Eromoto, R., Ksenofontov, L. T., et al. 2005, ApJ, 619, L163

Krymsky, G. F. 1977, Sov. Phys.-Doklady, 22, 327

Malkov, M. A. \& Drury, L. O’C. 2001, Reports Progr. Phys., 64, 429

Reynolds, S. P. 1998, ApJ, 493, 375

Vink, J. 2006, in The X-ray Universe, ESA SP-604 (ESA, ESREC), ed. A. Wilson, 1, 319

Völk, H. J., Berezhko, E. G., \& Ksenofontov, L. T. 2005, A\&A, 433, 229

Webb, G. M., Drury, L. O’C., \& Biermann, P. 1984, A\&A, 137, 185 